deposit. Eventually, Forbes, Ramsay, Whittlesey, Croll and others accumulated so much evidence that the 'Glacial Theory' which we take so much for granted today, became accepted by most geologists.

Not the least fascinating parts of the book are Cunningham's descriptions and assessments of the complex characters of Forbes and his friends and enemies. Forbes is described as "aloof, meticulous, cautious, exceedingly well-organised, the model of an inductive scientist", but generally disliked by his students. The ebullient Agassiz was "a man of genius, capable of making leaps in the ladder of his ideas, skipping rungs in a fashion that to Forbes would have been offensive, to arrive at a new and unfamiliar position from which he would then search for data to justify his whereabouts". Tyndall did "a great deal of peering through discoloured spectacles". But in extolling Forbes' achievements, Cunningham does not spare criticism where this is due.

The book is well researched and written, but the author is at such pains to develop his arguments that there is some unnecessary repetition. It is also rather

\section{Spinning stars}

\section{Martin Gaskell}

Pulsar Astronomy. By A. G. Lyne \& F. Graham-Smith. Cambridge University Press: 1990. Pp.274. £40, \$69.50.

IT wAS on 6 August 1967 that the first pulsar (PSR 1919+21) showed up on a chart-recording at Cambridge University. In the intervening 23 years, pulsars and neutron stars have taken their place in a very wide range of astrophysics. Pulsar Astronomy, the first monograph on the subject for more than a decade, provides an excellent introduction to what is known about pulsars as well as a discussion of some of the other areas of astrophysics influenced by pulsars.

In their preface, the authors say that their book will not be needed by the small band of pulsar specialists but that it is intended to be useful "perhaps to students entering the field and perhaps to those who enjoy an exciting story of discovery and of the application of basic physics to a realm unattainable in earthbound laboratories". The book is probably best suited for graduate students starting research on pulsars and for other scientists who want a quick overview of what is known about pulsars. It is about the right length and level for a one-term graduate-level introduction to the subject. Many parts do indeed tell "an exciting story of discovery" which will be appreciated by more general readers, even those with little scientific background.

Pulsar Astronomy is based in part on $\mathrm{Sir}$ longer than necessary because of the exhaustive details of Forbes' glacier measurements and the inclusion of some of his exciting mountaineering feats where glaciology was not much in evidence and which glaciologists (at least) could do without.

I would have liked a little more discussion at the end on the latest ideas on glacier structure and movement, and would have appreciated rather more detailed maps of Alpine glaciers and the areas of Norway that Forbes visited in 1851. The Swissair photographs of the Alps are essential to the text, which is compulsory reading for glaciologists, glacial geomorphologists and historians of science (many famous men of the mid-nineteenth century like Lyell, Murchison and Darwin are drawn into the narrative). It will also be of interest to Alpine mountaineers and to anyone who likes to read about the bitter controversies of past times and their adverse effects on those involved.

John H. McD. Whitaker was formerly Editor-inChief of Geology Today and is at 11 Guilford Road, Leicester LE2 2RD, UK.

Francis Graham-Smith's earlier monograph, Pulsars (Cambridge University Press, 1977). Less than half the original text and figures are retained, but for convenience I will refer to the 1977 book as the first edition and this volume as the new edition. In the 13 years between the two there has been much observational and theoretical progress, which is readily apparent if one compares the first and new editions. Almost every chapter has undergone major updating (and the order has been changed). There are now new chapters on binary and millisecond pulsars (now being discovered at a rapid rate in globular clusters) and X-ray binaries and bursters. Our knowledge of almost every aspect of pulsars has increased greatly, as has the known number of pulsars (incidentally, a useful feature of the book is the

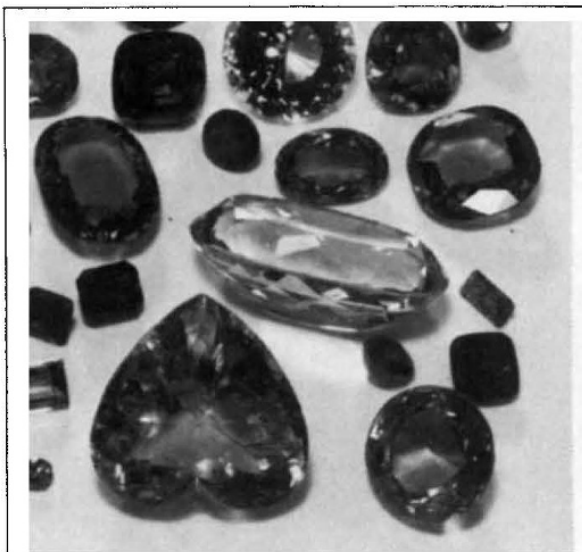

inclusion of an up-to-date catalogue of the 450-plus pulsars known up to March 1989).

The biggest theoretical advance since the first edition has probably been in addressing the question "why do pulsars pulse?"; the chapters discussing this problem have been totally rewritten. In the mid-1970s, there was no consensus as to whether the emission came from the magnetic poles of the pulsar or from near where the magnetic field was being swept round at almost the speed of light (the outer magnetosphere gap), but researchers were trying to explain the entire pulsed spectrum based on one or other of these origins. It is now accepted instead that there are two different origins: the highenergy photons come from the outer magnetosphere gap, whereas the radio beam originates directly over the magnetic pole. But even after 23 years of research, as Lyne and Graham-Smith point out, "the rotating lighthouse beam itself is the least understood of all the observable properties of pulsars".

The main deficiency is in the discussion of supernovae explosions (the main and perhaps only producers of pulsars), which contains quite a few inaccuracies and significant omissions. Rather surprisingly for a book published in 1990 , no mention is made of the fact that we now know that there are two types of supernovae lacking hydrogen, so-called type Ia and Ib supernovae. The reader could be left with the impression that low-mass stars barely more massive than the Sun might somehow become supernovae - the standard exploding binary white-dwarf model for type Ia supernovae is never mentioned. Neither is the fact that some type I supernovae are believed to come from young massive stars (and might even form pulsars). But these deficiencies are somewhat on the periphery of the main subject material of this book.

C. Martin Gaskell is in the Department of Astronomy, University of Michigan, Ann Arbor, Michigan 48109-1090, USA.

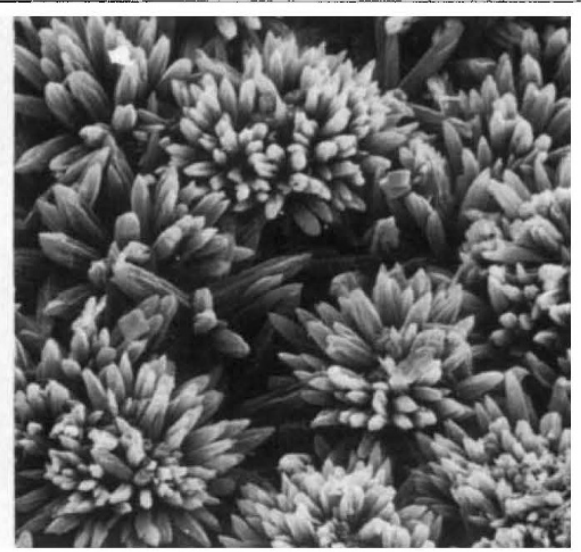

From macro to micro - the polished gems on the left and the kettle 'fur' $(\times 350)$ on the right are two examples of crystals taken from the book Crystals by lan F. Mercer. The book contains many more delightful pictures and reveals the secrets of crystals of various shapes, sizes and origins. Published by Natural History Museum Publications, London, price is $£ 4.95$ (pbk). 